\title{
ACTIVATION DU SODIUM PAR DES NEUTRONS DE FISSION, INTERCOMPARAISON DE MANNEQUINS ANTHROPOMORPHES DIFFÉRENTS*
}

\author{
D. DOUELLOU, MIle J. EVRARD**
}

(Manuscrit reçu le 5 décembre 1974)

\begin{abstract}
RESUME
Trois mannequins de types différents, ont été irradiés par des neutrons de fission. L'activation du sodium a été étudiée en fonction de l'orientation, en mesurant l'activité du ${ }^{24} \mathrm{Na}$ formé dans le mannequin entier, dans les compartiments correspondant aux parties anatomiques symétriques, dans les organes profonds. Ces résultats ont permis une comparaison entre les trois types de mannequins employés.
\end{abstract}

\section{ABSTRACT}

Three differents phantoms were irradiated by means of fission neutrons. The resultant sodium activation was studied by measuring ${ }^{24} \mathrm{Na}$ activity in whole body, in symetric parts of the body. and in deeper organs. These results gave the occasion of comparing the tested phantoms.

\section{INTRODUCTION}

Lorsque le corps humain est irradié par des neutrons, certains éléments qui le composent sont activés, en particulier: le soufre, le chlore et le sodium, selon les réactions :

$$
\begin{gathered}
{ }^{32} \mathrm{~S}(\mathrm{n}, \mathrm{p})^{32} \mathrm{P} \\
{ }^{37} \mathrm{Cl}(\mathrm{n}, \gamma){ }^{38} \mathrm{Cl} \\
{ }^{23} \mathrm{Na}(\mathrm{n}, \gamma)^{24} \mathrm{Na}
\end{gathered}
$$

${ }^{32} \mathrm{P}$ est un émetteur bêta ; la mesure de son activité nécessite une séparation chimique préalable. De plus, la réaction d'activation se produit principalement pour des neutrons d'énergie supérieure à $2,5 \mathrm{MeV}$.

\footnotetext{
(*) Ce travail a été effectué à l'occasion des études menées dans le cadre du « Groupe Dosimétrie CRAC » qui regroupe des équipes du DPr et du SESR.

(**) Commissariat à l'énergie atomique, Direction des Applications militaires, Centre de Valduc, Section de protection contre les rayonnements et Service Médical, BP 14, 21120 Is-sur-Tille.
} 
${ }^{38} \mathrm{Cl}$ est un émetteur bêta, gamma, mais sa période, $38 \mathrm{~min}$, gêne la détermination précise de son activité si la mesure est faite cinq à six heures après l'irradiation.

${ }^{24} \mathrm{Na}$ est un émetteur bêta, gamma, de période $15 \mathrm{~h}$. Pour chaque désintégration, il émet pratiquement deux photons, d'énergie respective $1,38 \mathrm{MeV}$ et $2,76 \mathrm{MeV}$. Du fait de la mobilité élevée de l'ion $\mathrm{Na}^{+}$, le ${ }^{24} \mathrm{Na}$ induit se met rapidement en équilibre avec la majorité du sodium corporel, de telle sorte qu'au bout d'une dizaine d'heures, 85 p. cent environ de l'activité due à cet isotope est répartie de manière à peu près homogène à travers l'organisme. La moitié du sodium osseux, soit environ 10 à $15 \mathrm{p}$. cent du sodium total ne s'échange qu'à très long terme avec le sodium circulant $[2,3,7]$. Cependant, des mesures faites sur des mulets [13] ont montré que l'on pouvait remplacer le corps de l'animal par un mannequin rempli avec une solution homogène d'un sel de sodium : l'activité spécifique du sang est alors proche de celle de la solution. Par suite, l'étalonnage des dispositifs de mesure peut s'effectuer à l'aide d'un mannequin contenant une solution connue de ${ }^{24} \mathrm{Na}[16]$.

On peut donc considérer le sodium corporel comme un dosimètre permanent, véritablement personnel, et dont l'activation rapidement mesurable peut constituer, quelquefois, l'un des rares critères auxquels on puisse se référer immédiatement. La validité de ce dosimètre n'est effective que dans la mesure où, connaissant l'activité du ${ }^{24} \mathrm{Na}$ induit dans l'organisme, on peut déterminer la dose reçue par le sujet [4]. En fait, pour une dose absorbée moyenne donnée, plusieurs paramètres sont susceptibles de modifier l'activation du sodium et sa mesure même. Le principal est évidemment la nonhomogénéité du champ de rayonnement atteignant l'organisme.

De plus, lorsque l'organisme est irradié par des neutrons, soit qu'ils proviennent directement de la source irradiante, soit qu'ils aient été diffusés entre-temps, ces neutrons perdent une partie de leur énergie en traversant les tissus. En un point donné de l'organisme, l'activation du sodium est fonction de facteurs multiples: la nature et l'épaisseur des tissus traversés, le spectre et la fluence neutronique en ce point, etc. Aussi, la position et l'orientation du sujet irradié, ses caractéristiques biométriques, le spectre énergétique des neutrons, leur fluence, sont-ils autant de facteurs qu'il est nécessaire de connaître mais qui sont rarement accessibles lors d'un accident réel.

Dans ce but, on procède le plus souvent à une reconstitution de l'accident dans laquelle un mannequin remplace la personne irradiée.

Idéalement, le mannequin utilisé devrait être identique au sujet, avoir le même poids, la même taille, l'équivalent d'un squelette et d'organes, être réalisé avec des matériaux ayant une composition chimique voisine de celle des tissus, etc.

Les mannequins employés en pratique sont loin de remplir ces exigences. Leurs caractéristiques morphologiques se rapprochent en général de celles de «l'homme standard » défini par la C.I.P.R., ce qui ne permet pas de tenir compte des différences individuelles. La forme du corps elle-même 
n'est reproduite parfois que grossièrement ; enfin, dans les mannequins les plus usités, il n'y a pas l'équivalent d'un squelette.

Que ce soit pour mesurer l'activité du ${ }^{24} \mathrm{Na}$ ou pour en étudier l'induction dans le corps sous irradiation, le choix d'un type donné de mannequin est de première importance. Les résultats et leur mode d'interprétation peuvent dépendre largement de ce choix.

Le but de ce travail est de contribuer à établir des critères de comparaison entre les divers types de mannequins les plus employés actuellement.

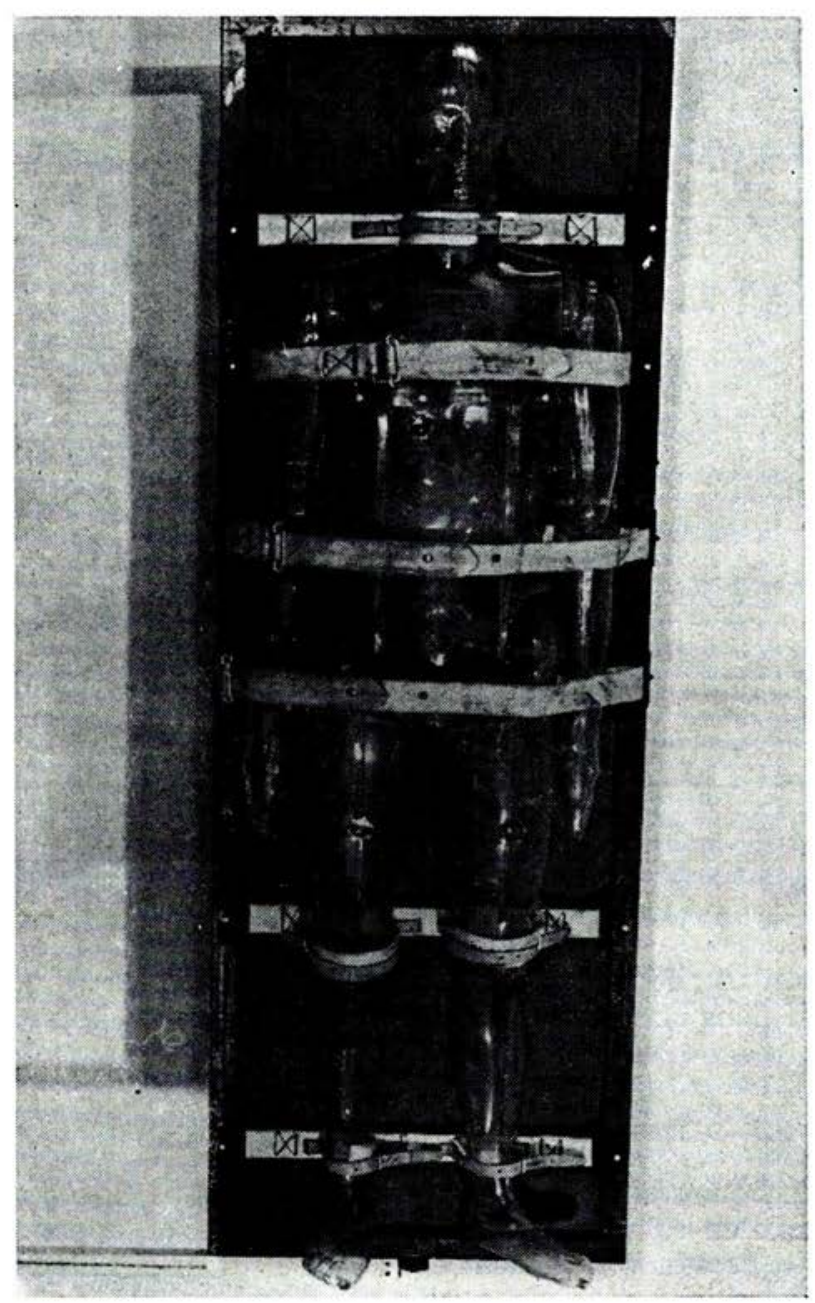

FIG. 1. - Mannequin ReMCAL 


\section{I. - MATÉRIEL ET MÉTHODES}

\section{A) ManNequins}

REMCAL : Mannequin anthropomorphe avec organes, constitué de compartiments étanches (Tableau I et fig. 1).

BOMAB : Mannequin constitué de compartiments étanches en polyéthylène, de forme cylindrique ou ellipsoïdale, sans organe (Tableau I et fig. 2).

TABLEAU I

CARACTERISTIQUES DES MANNEQUINS

\begin{tabular}{|c|c|}
\hline REMCAL & BOMAB \\
\hline $\begin{array}{l}\text { Constructeur : } \\
\text { Matière } \\
\begin{array}{l}\text { : Butyrate d'acétate de } \\
\text { cellulose }\end{array}\end{array}$ & $\begin{array}{l}\text { Constructeur: } \\
\text { Matière } \quad \text { : Polypropylène }\end{array}$ \\
\hline Volumes des compartiments $\left(\mathrm{cm}^{3}\right)$ : & Volume des compartiments $\left(\mathrm{cm}^{3}\right)$ \\
\hline Tête +thorax . . . . . . $9750 \mp 30$ & 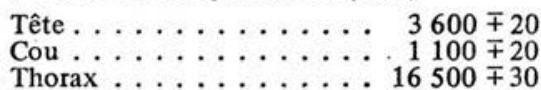 \\
\hline$\ldots \ldots \ldots 14150 \mp 30$ & Bassin $\ldots \ldots \ldots \ldots 10200 \mp 30$ \\
\hline $1700 \mp 20$ & $\ldots \ldots \ldots 1650 \mp 20$ \\
\hline Bras droit $\ldots \ldots \ldots \ldots$ & Bras droit $\ldots \ldots \ldots \ldots$ \\
\hline Avant-bras gauche $\ldots \ldots$ & Avant-bras gauche $\ldots \ldots \ldots$ \\
\hline Avrant-bras droite $\ldots \ldots$ & Avant-bras droit $\ldots \ldots \ldots$ \\
\hline Cuisse gauche $\ldots \ldots \ldots$ & Cuisse gauche $\ldots \ldots \ldots$ \\
\hline Cuisse droite. . . . . . . . . & Cuisse droite $\ldots \ldots \ldots \ldots$ \\
\hline Jambe gauche $\ldots \ldots \ldots$ & Jambe gauche $\ldots \ldots \ldots$ \\
\hline Jambe droite. . . . . . . & Jambe droite $\ldots \ldots \ldots \ldots$ \\
\hline Poumon gauche supérieur . . & \\
\hline Poumon gauche inférieur ... & \\
\hline Poumon droit supérieur . . . & \\
\hline Poumon droit inférieur. . . . & \\
\hline Foie $\ldots \ldots \ldots \ldots \ldots$ & \\
\hline Cœur $\ldots \ldots \ldots \ldots \ldots$ & \\
\hline Rate $\ldots \ldots \ldots \ldots \ldots$ & \\
\hline Rein droit $\ldots \ldots \ldots \ldots$ & \\
\hline Rein gauche $\ldots \ldots \ldots$ & \\
\hline Volume total $\left(\mathrm{cm}^{3}\right) \ldots \ldots 54130$ & Volume total $\left(\mathrm{cm}^{3}\right)$ \\
\hline
\end{tabular}

Plastinaute : Mannequin anthropomorphe plein, constitué d'un squelette enchassé dans une matière équivalente aux tissus vis-à-vis des neutrons (fig. 3).

\section{B) IRRADIATION (DISPOSITIF CRAC)}

Les mannequins sont irradiés par des neutrons de fission produits par la divergence d'un solution d'uranium enrichi. Une description précise de l'installation et de son fonotionnement est donnée dans la référence [11]. Les conditions de criticité sont obtenues en remplissant une cuve cylindrique jusqu'à la hauteur critique. 


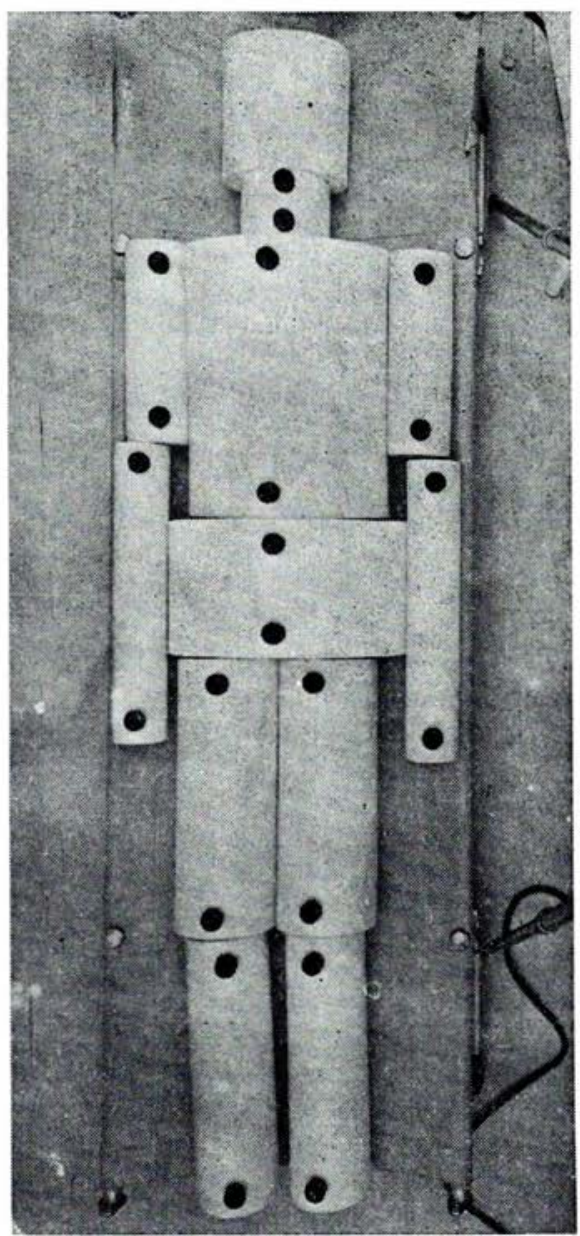

Fig. 2. - Mannequin BoмАB

\section{Mise en Place DES MANNEQUiNS}

Les mannequins, à l'exception du Plastinaute, sont fixés sur un cadre métallique à l'aide de sangles. Le cadre est suspendu à une barre horizontale soutenue elle-même par deux barres verticales, de telle sorte que les pieds du mannequin reposent sur le sol. La position du mannequin est repérée à l'aide d'un système de coordonnées reproduit sur la figure 4.

Quatre positions ont été étudiées :

Positions $N^{\circ} 1$ et 2 : Plastinaute 7B +100 , Remcal, Bomab 7B -100 . 


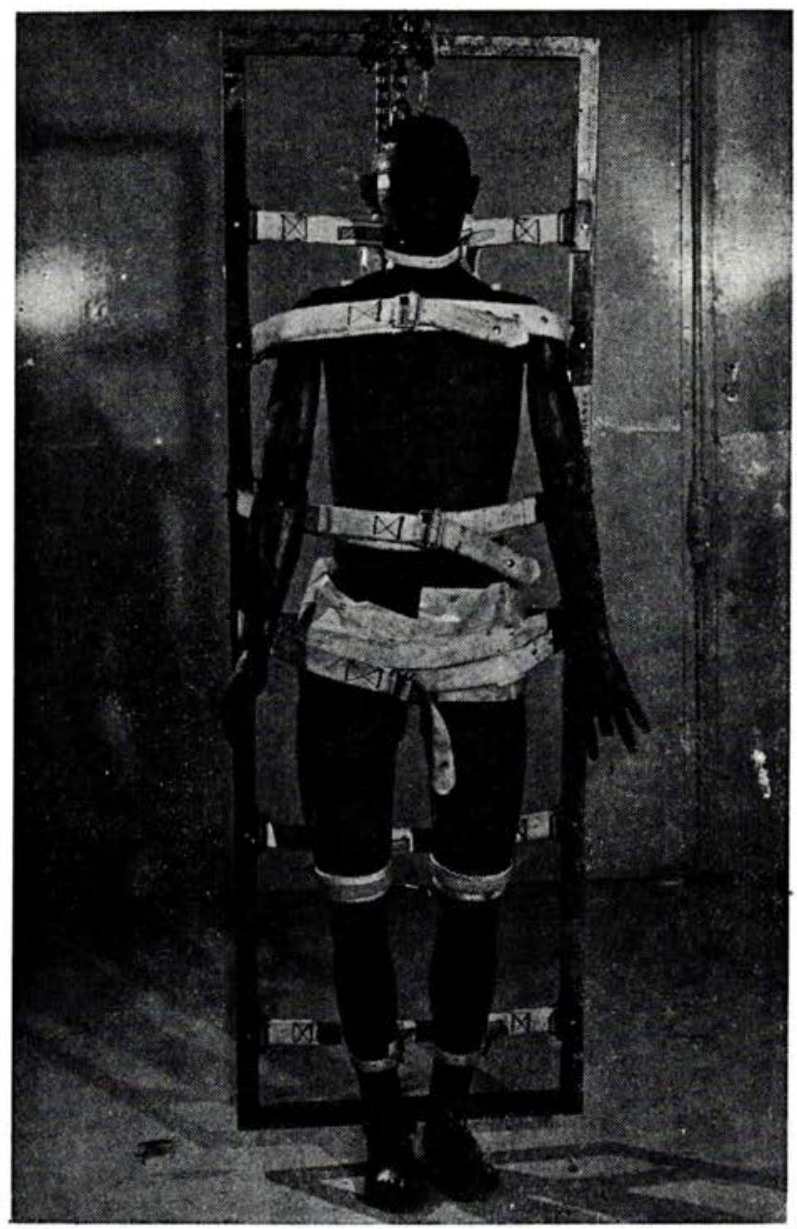

Fig. 3. - Mannequin Plastinaute

Lorsque le mannequin fait un angle avec la direction du rayonnement primaire, le côté le plus proche de la source d'irradiation est le côté droit, sauf pour $\gamma=3 \pi / 2$.

L'orientation du mannequin est repérée par l'angle alpha entre son axe transversal droit-gauche et la normale à la direction du rayonnement primaire. Cet angle varie dans le sens trigonométrique. Le mannequin est placé de manière correcte lorsque le point le plus proche de la source est à la verticale du point choisi. $-0,25$.

Positions $N^{\circ} 3$ et 4 : Remcal 6,35 S - 0,25, Plastinaute 5,25 R 
B $\quad S \quad P$

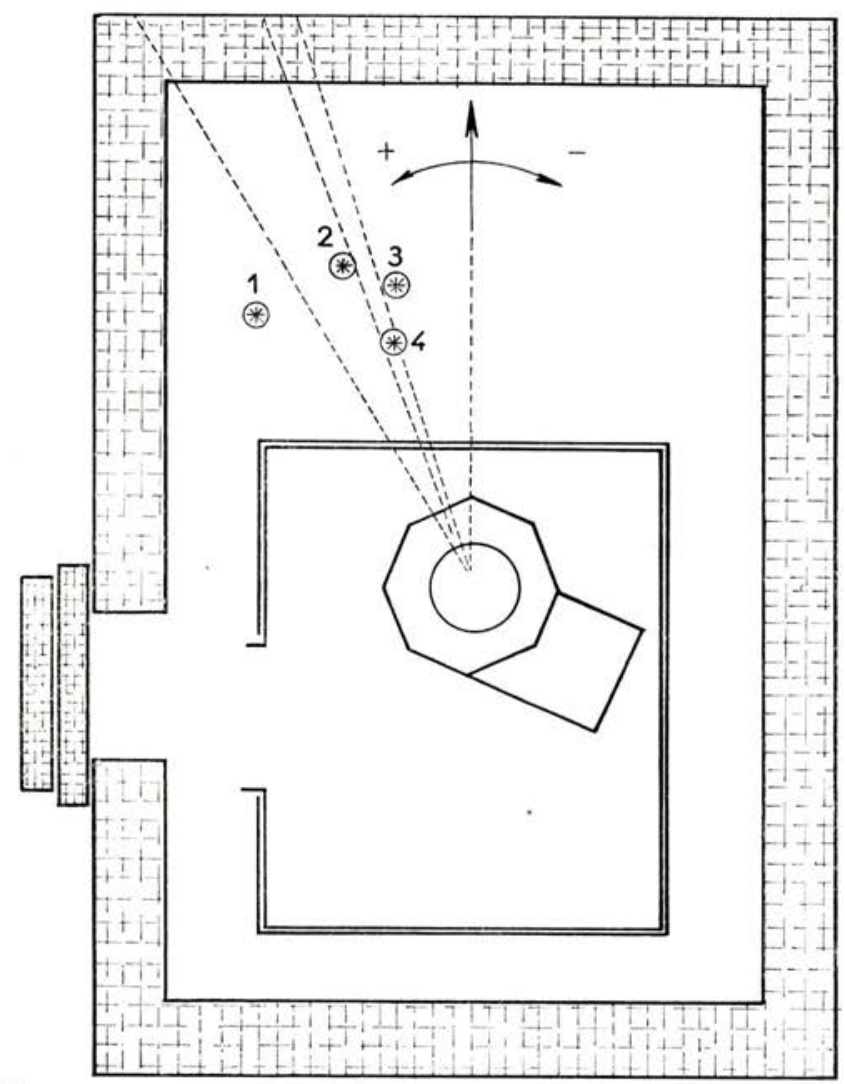

$1 \mathrm{~m}$

FIG. 4. - Enceinte d'irradiation, positions occupées par les mannequins

Le mannequin est en place lorsque son axe vertical passe par le point choisi. Le côté droit est le plus proche de la source. L'orientation du mannequin est repérée comme précédemment.

\section{C) SOLUTION IRRADIÉE}

Tous les compartiments étanches des mannequins BomaB et REMCAL sont remplis avec une solution aqueuse de chlorure de sodium d'un titre pondéral théorique de $0,094 \mathrm{p}$. cent de sodium (teneur théorique en sodium de la matière équivalente aux tissus composant le Plastinaute).

Le titre réel est ensuite contrôlé à l'aide d'un photomètre de flamme à absorption atomique PERKIN-ELMER type 303. Cet appareil est étalonné à l'aide de solutions aqueuses de $\mathrm{NaCl}$ Merck qualité supra-pure. 
D) MEsure DE L'ACTIVIté du ${ }^{24} \mathrm{Na}$

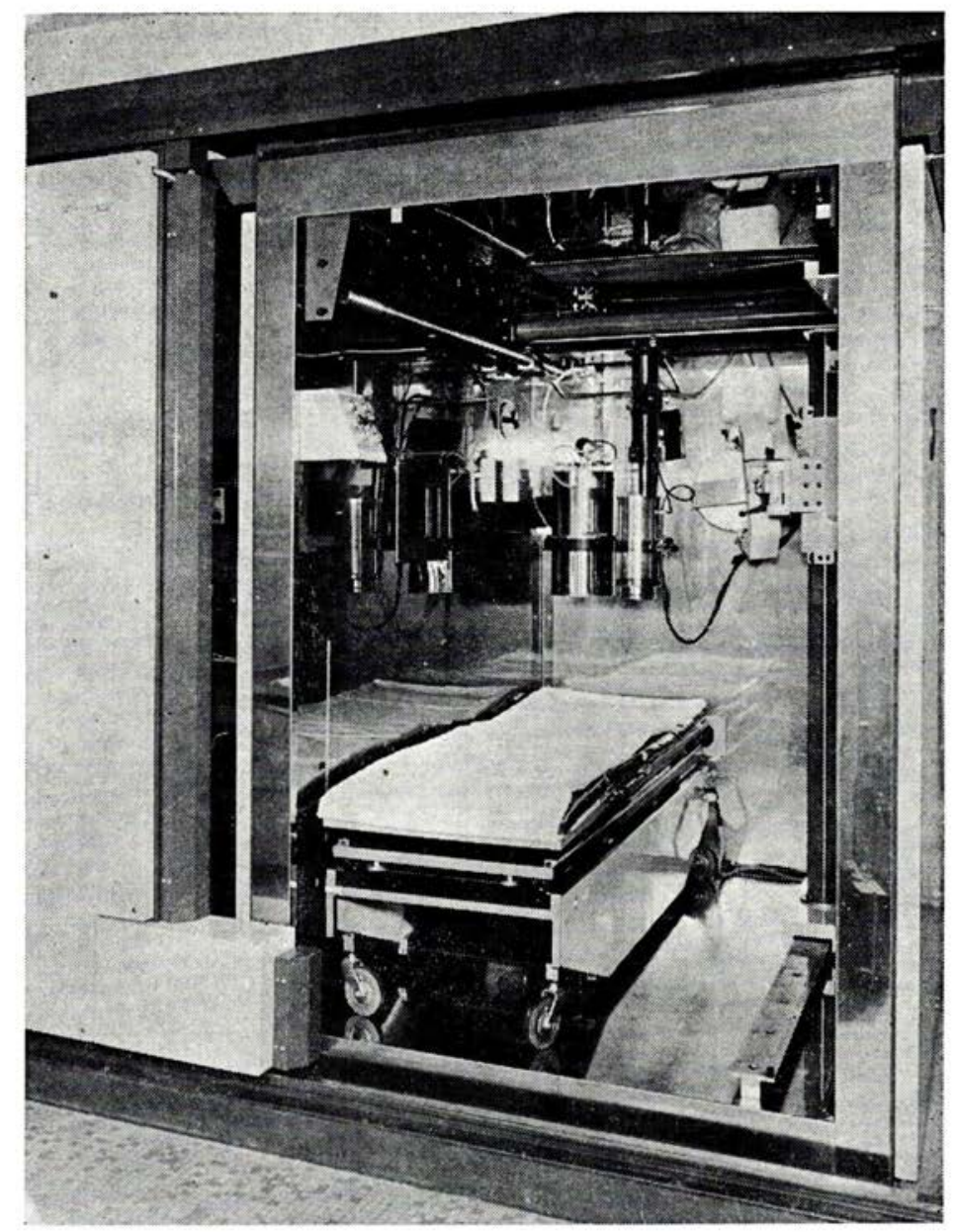

Fig. 5. - Ensemble de mesure

1) Appareillage : L'ensemble de mesure (fig. 5) comprend : d'épaisseur,

- une chambre blindée constituée par des blocs de plomb de $10 \mathrm{~cm}$

- une première sonde à cristal d'iodure de sodium activé au thallium, $44 \mathrm{~cm} \times 51 \mathrm{~cm}$, équipé d'un photomultiplicateur RCA,

— une seconde sonde à cristal NaI $(\mathrm{Tl}) 203 \mathrm{~cm} \times 77 \mathrm{~cm}$, associée à quatre photomultiplicateurs. 
Ces deux détecteurs sont reliés à un sélecteur d'amplitude à 400 canaux INTERTECHNIQUE. Les résultats sont enregistrés à l'aide d'une perforatrice à ruban.

\section{2) Réglages}

Le sélecteur est réglé à $20 \mathrm{keV} /$ canal entre 0,1 et $4 \mathrm{MeV}$. Le temps de comptage est de 15 min pour chaque mesure, temps réel, compte tenu des corrections de bruit de fond, de l'activité des supports (mannequins avant irradiation, boîtes de Petri, selon les cas) et du temps mort éventuellement.

\section{3) Géométrie}

a) Mesure de l'activité d'un mannequin entier

Le mannequin est allongé sur un brancard, le cristal de la sonde 1 à $1,10 \mathrm{~m}$ du point situé à mi-chemin entre l'ombilic et la fourchette sternale, dans l'axe sagittal médian du mannequin.

\section{b) Activité volumique de la solution}

Celle-ci est mesurée à l'aide de 1a sonde 2. Des aliquotes de $25 \mathrm{~cm}^{3}$ correspondant chacune à un compartiment différent (du REMCAL ou du BOMAB), sont transférées dans des boîtes de Petri (diamètre $50 \mathrm{~mm}$ ). Celles-ci sont placées dans l'axe principal de la sonde, bords au contact.

\section{4) Exploitation des résultats}

a) Activité volumique de la solution contenue dans chaque compartiment (REMCAL et BOMAB)

Les spectres relevés par le sélecteur sont mis sous forme de rubans perforés et traités en temps différé par un ordinateur Digital type PDP8. Pour un spectre quelconque, les données sont comparées à celles fournies par un ruban perforé obtenu dans les mêmes conditions à partir d'une solution étalonnée de ${ }^{24} \mathrm{Na}$ fournie périodiquement par le LMR, Saclay.

Le calcul est conduit en trois parties : Deux estimations sont faites en comparant, entre le spectre mesure et le spectre étalon, le nombre de chocs pour les bandes d'énergies $1,37 \mp 0,14 \mathrm{MeV}$ et 2,76 $\mp 0,14 \mathrm{MeV}$. Une troisième est réalisée en ajustant tous les points du spectre étalon aux points du spectre de mesure, par la méthode des moindres carrés.

Après avoir vérifié la concordance des trois valeurs, on considère finalement leur moyenne arithmétique et on effectue une correction pour tenir compte de la décroissance radioactive du ${ }^{24} \mathrm{Na}$. L'activité de celui-ci est ramenée à la fin de l'irradiation.

\section{b) L'activité totale du mannequin Remcal}

Elle est obtenue en multipliant l'activité volumique, obtenue de la manière précédente, par le volume du compartiment correspondant, puis en faisant la somme de ces valeurs. 
Pour les bandes d'énergie $0,1--4 \mathrm{MeV} .1,37 \mp 0,14 \mathrm{MeV}, 2,76 \mp$ $0,14 \mathrm{MeV}$, le nombre de chocs donnés par le Plastinaute, par l'intermédiaire de la sonde 1, est comparé avec les valeurs obtenues avec le REMCAL. L'activité du Plastinaute est donnée par la moyenne de ces trois évaluations, par rapport à l'activité du REMCAL.

c) Précision

L'activité réelle de chaque partie du REMCAL est donnée dans un intervalle de 10 à 15 p. cent de la valeur indiquée. Cette estimation tient compte des erreurs systématiques (pesées, mesures de volume, pipetages, etc.) et des erreurs statistiques sur les taux de comptage.

\section{E) PROTOCOLE OPÉRATOIRE}

Deux mannequins sont irradiés simultanément au cours de chaque expérience (Plastinaute et Bomab ou RemCAL). Le mode opératoire général est schématisé dans le tableau II.

TABLEAU II

PROTOCOLE OPERATOIRE

\begin{tabular}{|c|c|}
\hline DATES & OPERATIONS \\
\hline $\mathrm{J}-3$ & $\begin{array}{l}\text { - Préparation de la solution de } \mathrm{NaCl}: 60 \text { litres à } 0,094 \text { pour cent } \\
\text { de } \mathrm{Na} \text { en poids. }\end{array}$ \\
\hline $\mathrm{J}-2$ & $\begin{array}{l}\text { - Remplissage du mannequin. Prélèvements pour mesure de la concen- } \\
\text { tration de Na (l'un est fait avant remplissage, l'autre sur le volume } \\
\text { restant après remplissage). } \\
\text { - Contrôle de l'étanchéité du mannequin. } \\
\text { Colmatages éventuels. }\end{array}$ \\
\hline $\mathbf{J}-1$ & $\begin{array}{l}\text { - Mesure de l'activité du mannequin rempli. } \\
\text { - Vérification des deux sondes de mesure (résolution, réglages des } \\
\text { PM, etc.). } \\
\text { - Mise en place des mannequins dans l'enceinte d'irradiation. }\end{array}$ \\
\hline$\stackrel{\mathbf{J}}{\mathrm{J}}+1$ & $\begin{array}{l}\text { - Mesures alternatives de l'activité totale de chaque mannequin à } \\
\text { l'aide de la sonde } n^{0} 1 .\end{array}$ \\
\hline $\mathrm{J}+2$ & $\begin{array}{l}\text { - Prélèvement de solution dans chaque compartiment du REMCAL } \\
\text { ou du BOMAB, et mesure d'activité (sonde } n^{0} 2 \text { ). }\end{array}$ \\
\hline $\begin{array}{l}\mathrm{J}+3 \\
\mathrm{~J}+4\end{array}$ & $\begin{array}{l}\text { - Mesure de l'activité totale du Plastinaute toutes les } 5 \text { heures. } \\
\text { - Exploitation des mesures. }\end{array}$ \\
\hline
\end{tabular}

\section{RESULTATS}

\section{A. ACtivité totale INDUite DANS LES TROIS MANNEQUiNS}

Les activités sont exprimées en $\mu \mathrm{Ci}$ pour $10^{18}$ fissions, et sont normalisées, quand il y a lieu, pour un titre pondéral de 0,094 p. cent en sodium. 
En ce qui concerne le Вомав, une correction supplémentaire est introduite pour ramener le volume de solution irradiée au volume du REMCAL, soit $54130 \mathrm{~cm}^{3}$.

Les valeurs obtenues sont consignées dans le tableau III.

TABLEAU III

ACTIVITE TOTALE DU ${ }^{24} \mathrm{Na}$ INDUIT DANS LES MANNEQUINS

\begin{tabular}{|c|c|c|c|c|}
\hline & $\alpha$ & PLASTINAUTE & REMCAL & BOMAB \\
\cline { 2 - 5 } Positions & 0 & $170^{*}$ & 84 & 95 \\
1 et 2 & $\pi / 4$ & - & $81^{* *}$ & 98 \\
& $\pi / 2$ & - & 70 & 86 \\
\hline & & & 100 & - \\
Positions & $\pi / 3$ & 248 & 102 & - \\
3 et 4 & $\pi 2$ & 236 & 83 & - \\
\hline
\end{tabular}

* Moyenne obtenue à la suite des expériences CRAC 14-17-19.

** Valeur obtenue pour CRAC 21. L'activité volumique du bassin a été supposée égale à celle du compartiment tête et thorax (défaut d'étanchéité).

Les résultats concernant le BomaB et le REMCAL (position 1) sont en bonne concordance. Une rotation de $45^{\circ}$ ou de $60^{\circ}$ ne semble pas modifier significativement l'activité totale induite par rapport à la position de face. Lorsque le mannequin est de profil, la diminition d'activité est de $15 \mathrm{p}$. cent pour l'un, 16 p. cent pour l'autre. En position 2, la différence face-profil pour le Plastinaute est de 20 p. cent, elle est de 17 p. cent pour le REMCAL en position 3.

Ces résultats sont en bon accord avec ceux trouvés par ailleurs [6, 13, 14]. Il paraît, cependant, surprenant que l'activité totale ne varie pas, que le mannequin soit de face ou tourné de $45^{\circ}$ ou $60^{\circ}$.

L'activité du Plastinaute est environ le double de l'activité mesurée avec les autres mannequins, dans des conditions analogues d'irradiation. Les résultats des analyses effectuées par le Département de Protection sur les constituants du Plastinaute expliquent cet écart :

matière équivalente aux tissus mous : $1,1 \mathrm{mg} \mathrm{de} \mathrm{Na} / \mathrm{g}$ de matière ; squelette : 9,25 mg de $\mathrm{Na} / \mathrm{g}$ d'os.

Le squelette ayant une charge sodique très supérieure, l'activité du ${ }^{24} \mathrm{Na}$ après irradiation est majorée en conséquence par rapport aux deux autres mannequins qui ne comportent pas de squelette.

B. Activation du SODIUM Dans les parties homologues DU REMCAL et DU BOMAB

La probabilité de capture neutronique par le sodium corporel a tout d'abord été donnée par HuRst et Ritchie [8]. Ces calculs ont été repris voL. $9-\mathrm{N}^{\circ} 4$ 
par AUXIER, SANDERS et SNYDER [12, 13], en assimilant le corps humain à un cylindre de $30 \mathrm{~cm}$ de diamètre et de $60 \mathrm{~cm}$ de haut. Ces résultats diffèrent de ceux de MCGUIRE [9] qui utilise un modèle plus élaboré, composé de cylindres et d'ellipsoïdes, très proche du mannequin BoMAB. Une vérification expérimentale de cette étude basée sur la méthode des multisphères [6] a abouti à une concordance raisonnable. L'emploi d'un BOMAB comme référence expérimentale serait donc justifié, à la condition qu'il n'y ait pas de différences trop grandes entre l'activation du sodium dans le mannequin et dans un corps humain réel. Sur le seul plan morphologique, un mannequin Вомав est très différent d'un homme; or, les calculs des coefficients d'activation font intervenir la section de l'individu interceptant le flux [10]. Pour les membres par exemple, cette section est différente selon que l'individu est de face ou de profil par rapport au flux, alors qu'elle reste la même pour le Bомав puisque les membres sont remplacés par des cylindres. Les mannequins type RemCAL et Plastinaute par contre, reproduisent bien la forme du corps humain.

Nous avons donc cherché à comparer entre elles les valeurs de l'activité spécifique du ${ }^{24} \mathrm{Na}$ dans les compartiments homologues du REMCAL et du BомАВ, ceci pour la même position et la même orientation par rapport à la direction du flux direct. Les résultats sont reportés dans le tableau IV.

Pour les irradiations de face, $a$ désigne l'activité spécifique de la solution, ramenée à la fin de l'irradiation, exprimée en $\mu \mathrm{Ci}$ de ${ }^{24} \mathrm{Na} / \mathrm{g}$ de sodium, pour $10^{8}$ fissions. Les autres colonnes donnent l'écart sur $a$, en pourcentage, par rapport à la valeur obtenue pour la position de face.

Lorsque le Remcal est placé de profil $\left(\alpha=90^{\circ}\right)$ les écarts observés sur l'activation des parties sont égaux à quelques p. cent près, que le mannequin occupe la position 2 ou la position 3 .

En ce qui concerne les écarts relatifs à l'orientation $\alpha=90^{\circ}$, on remarque, pour les deux mannequins, une bonne concordance des valeurs, qui est de l'ordre de 12 à $15 \mathrm{p}$. cent en moyenne pour les compartiments de droite. Il faut remarquer que l'avant-bras droit masque en partie la cuisse droite lorsque le mannequin est de profil. L'activité spécifique dans ce compartiment est donc inférieure à celle mesurée dans les autres parties situées à la droite du mannequin. Les parties gauches du BomAB présentent par contre des écarts qui diffèrent sensiblement de ceux observés pour les compartiments homologues du REMCAL. Comme pour $\alpha=90^{\circ}$ le mannequin tout entier fait écran pour les parties gauches, l'absorption du flux semble s'effectuer différemment pour le REMCAL et le BOMAB. Ceci pourrait faire préférer l'emploi d'un REMCAL, car ce type de mannequin est beaucoup plus proche de la morphologie humaine que ne l'est le mannequin Вомав.

Mais d'autres facteurs pourraient expliquer tout aussi bien ces fluctuations. Lorsque l'irradiation se produit, le mannequin est traversé par les neutrons du flux direct et aussi par des neutrons secondaires réfléchis ou diffusés par les murs, le sol, les objets présents dans la salle, etc., et selon des proportions variables d'une irradiation à l'autre, car la configuration 
TABLEAU IV

\begin{tabular}{|c|c|c|c|c|c|c|c|c|}
\hline & \multicolumn{8}{|c|}{$\begin{array}{l}\text { ACTIVITE SPECIFIOUE EN }{ }^{24} \mathrm{Na} \text { DE LA SOLUTION CONTENUB } \\
\text { DANS LES COMPARTIMENTS DU REMCAL ET DU BOMAB }\end{array}$} \\
\hline & \multicolumn{5}{|c|}{ MANNEQUIN REMCAL } & \multirow{2}{*}{\multicolumn{3}{|c|}{$\begin{array}{c}\text { MANNEQUIN BOMAB } \\
\text { Position } \mathrm{N}^{\circ} 1\end{array}$}} \\
\hline & \multicolumn{2}{|c|}{ Position $\mathrm{N}^{\circ} 1$} & \multicolumn{3}{|c|}{ Position $\mathrm{N}^{\circ} 3$} & & & \\
\hline & $\underset{\substack{\alpha=0^{\circ} \\
a}}{\text { CRAC } 17}$ & $\begin{array}{l}\text { CRAC } 20 \\
\alpha=90^{\circ} \\
\text { Ecart } \%\end{array}$ & $\underset{\substack{\alpha=0^{\circ} \\
a}}{\operatorname{CRAC} 12}$ & $\begin{array}{l}\text { CRAC 24 } \\
\alpha=60^{\circ *} \\
\text { Ecart \% }\end{array}$ & $\begin{array}{l}\text { CRAC } 30 \\
\alpha=90^{\circ} \\
\text { Ecart } \%\end{array}$ & $\begin{array}{c}\text { CRAC } 19 \\
\alpha=0^{\circ} \\
\text { Ecart } \%\end{array}$ & $\begin{array}{l}\text { CRAC } 29 \\
\alpha=45^{\circ} \\
\text { Ecart } \%\end{array}$ & $\begin{array}{c}\text { CRAC } 14 \\
\alpha=90^{\circ} 3 \pi / 4 \\
\text { Ecart \% }\end{array}$ \\
\hline Têtie & - & - & - & - & - & 1,90 & +05 & -04 \\
\hline Cou & - & - & - & - & - & 1,88 & +07 & 0 \\
\hline Thorax** & 1,69 & -5 & 1,94 & +11 & -07 & 1,59 & 0 & -19 \\
\hline Bassin & 1,57 & -23 & 1,85 & -06 & -26 & 1,55 & -03 & -23 \\
\hline Foie & 2,06 & -41 & 2,62 & -09 & -42 & - & - & - \\
\hline Cour & 2,12 & -57 & 2,47 & -33 & -57 & - & - & - \\
\hline Rate & 1,90 & -50 & 2,27 & -38 & -52 & - & - & - \\
\hline Bras droit & 1,73 & +15 & 2,02 & +41 & +18 & 1,96 & +17 & +12 \\
\hline Avant-bras droit & 1,51 & +15 & 1,77 & +35 & +14 & 1,96 & +12 & +13 \\
\hline Cuisse droite & 1,71 & +02 & 2,08 & +26 & +02 & 1,84 & +09 & -02 \\
\hline Jambe droite & 1,53 & +04 & 1,75 & +35 & +10 & 1,90 & +10 & +08 \\
\hline Poumon droit supérieur & 1,61 & -22 & 1,93 & +06 & -22 & - & - & - \\
\hline Poumon droit inférieur & 1,51 & -17 & 1,77 & +12 & -13 & - & - & - \\
\hline Rein droit & 1,10 & +22 & 1,20 & +36 & +28 & - & - & - \\
\hline Bras gauche & 1,78 & -35 & 1,96 & -27 & -38 & 1,84 & -08 & -33 \\
\hline Avant-bras gauche & 1,49 & -26 & 1,62 & -12 & -28 & 1,77 & -02 & -35 \\
\hline Cuisse gauche & 1,69 & -28 & 2,04 & -07 & -36 & 1,73 & -03 & -31 \\
\hline Jambe gauche & 1,49 & -09 & 1,75 & +14 & -17 & 1.63 & +18 & -27 \\
\hline Poumon gauche supérieur & 1,57 & -39 & 1,73 & -28 & -43 & - & - & - \\
\hline Poumon gauche inférieur & 1,51 & -43 & 1,69 & -27 & -46 & - & - & - \\
\hline Rein gauche & 1,14 & -03 & 1,22 & +02 & -07 & - & - & - \\
\hline
\end{tabular}

- La position exacte est 6,20 S - 0,25 au licu de 6,35 S-0,25

** Englobe tête et cou pour le REMCAL

a Activité spécifique ramenée à la fin de l'irradiation et exprimée en $\mu \mathrm{Ci}$ de ${ }^{24} \mathrm{Na} / \mathrm{g} \mathrm{de}{ }^{23} \mathrm{Na}$ et pour $10^{15}$ fissions.

topographique de l'enceinte n'est jamais rigoureusement la même (emplacements de détecteurs divers, écrans de plomb, matériel de levage, etc.). En supposant que cette fraction n'est pas trop importante vis-à-vis de la fluence des neutrons primaires, l'irradiation d'un mannequin, placé de face, conduit à une activité induite imputable surtout au flux direct. Si le mannequin est placé de profil, l'activation du sodium contenu dans les compartiments qui font face à la source, et ceux de grande capacité (bassin, thorax) est due encore principalement au flux primaire: les variations de l'activité spécifique par rapport à l'orientation de face sont reproductibles pour les deux mannequins, qu'ils soient placés en position 2 ou en position 3 . La part prise par les neutrons diffusés dans l'activation du sodium peut être soulignée dans le cas présent, si l'on compare nos résultats avec ceux de J.C. BAILEY [1]. Cet auteur a réalisé des irradiations de mannequins (type BOMAB) placés de face et de profil, mais surélevés par rapport au sol et hors de toute enceinte. 
En effectuant, pour les irradiations de profil, le rapport des activités des parties homologues, on obtient les valeurs suivantes :

\section{J.C. Bailey}

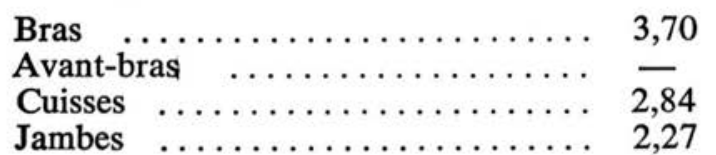

Présente étude

$$
\begin{aligned}
& 1,52 \\
& 1,59 \\
& 1,34 \\
& 1,27
\end{aligned}
$$

Les causes de cet écart entre nos résultats et ceux de BaILEY sont doubles. Le spectre neutronique est différent dans les deux cas, mais si l'on se réfère au modèle de SNYDER, on voit que la probabilité d'activation est sensiblement constante pour les neutrons dont l'énergie est supérieure à quelques $\mathrm{eV}$. Le fait que les spectres neutroniques soient différents ne peut donc expliquer à lui seul l'importance de cet écart. Par contre, dans les conditions d'irradiation rapportées par J.C. BAILEY, la fraction de neutrons diffusés, qui traversent le mannequin, est pratiquement nulle.

L'activation du sodium est alors due essentiellement au flux neutronique direct et, lorsque le mannequin est orienté de profil, l'activité induite dans les parties en regard de la source est bien supérieure à celle des parties masquées par le mannequin lui-même. Cela montre, en substance, l'importance des neutrons diffusés au cours des irradiations de type CRAC où il n'est pas toujours aisé de mettre en évidence l'influence du flux neutronique direct. En effet, l'activation du sodium est induite par les neutrons du flux direct (dont la direction d'incidence est déterminée) et par les neutrons diffusés ou réfléchis (dont les directions d'incidence par rapport au mannequin peuvent être quelconques). Le mannequin fait office d'écran vis-à-vis de ses compartiments propres qui ne sont pas en vue directe de la source : dans ces compartiments, l'activation du sodium par les neutrons diffusés est donc relativement plus importante. Cet effet devient maximal lorsque le mannequin est de profil et dépend de sa morphologie : les écarts observés diffèrent pour le REMCAL et le BOMAB placés de profil.

Pour les positions intermédiaires $\left(\alpha=45^{\circ}, \alpha=60^{\circ}\right)$, on observe effectivement une différence entre les variations de l'activité spécifique du sodium contenu dans les compartiments symétriques (droit-gauche). Par contre, les valeurs de l'activité spécifique de la solution contenue dans les compartiments axiaux de grande capacité (bassin, thorax) diffèrent peu des valeurs obtenues lorsque le mannequin est de face. L'activité globale induite reste sensiblement la même car les augmentations compensent les diminutions.

\section{ACtivation DU SODIUM DANS LES ORGANES PROFONDS}

Les données sont rassemblées sur la figure 6. Les compartiments du REMCAL simulent des organes humains et, en conséquence, ils ne sont ni placés de manière symétrique par rapport à l'axe longitudinal du corps, ni disposés à des niveaux d'égale épaisseur. En imaginant le mannequin tournant sur lui-même devant un repère fixe, l'épaisseur de tissu entre un organe 
comme le cœur et ce repère, n'est jamais la même au cours d'une rotation complète. Il s'ensuit que la quantité de ${ }^{24} \mathrm{Na}$ induit au niveau des organes profonds, et donc la dose reçue par ceux-ci, dépendent étroitement de l'orientation.

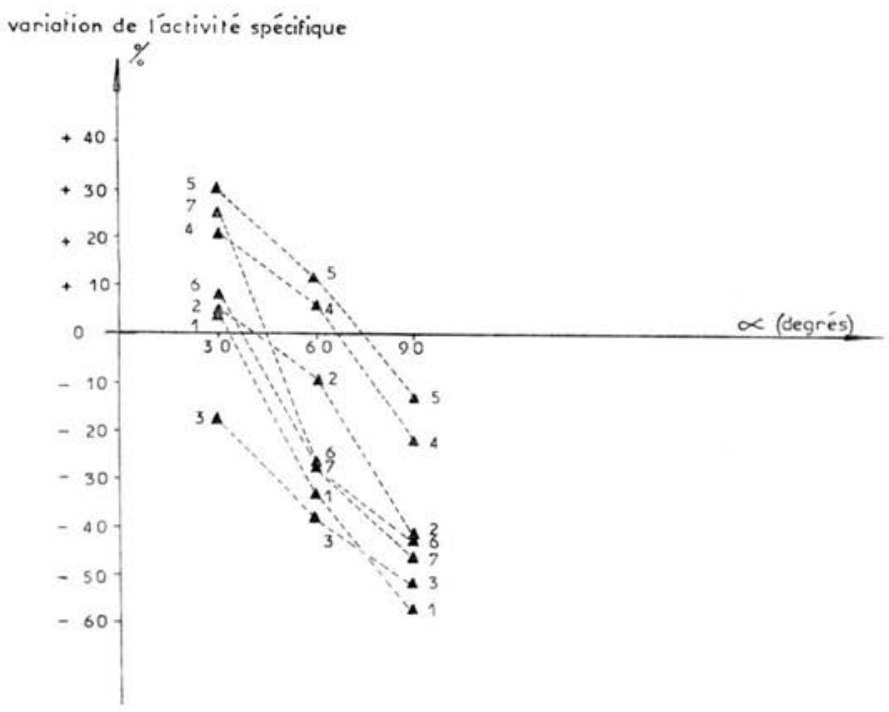

FIG. 6. - Activation relative des organes du REMCAL en fonction de l'orientation (position 3): 1: Cœur ; 2: Foie ; 3: Rate ; 4: Poumon droit, partie sup.; 5: Poumon droit, partie inf.; 6: Poumon gauche, partie sup.,; 7: Poumon gauche, partie inf.

Nous nous sommes bornés, pour notre part, à recueillir quelques valeurs $\left(0^{\circ} \leqslant \alpha \leqslant 90^{\circ}\right)$ obtenues avec le Remcal. Celui-ci ne comporte pas de squelette, dont l'influence est certainement à considérer : ces résultats ne peuvent donc être que des indications.

\section{CONCLUSIONS}

Les modalités d'emploi de mannequins anthropomorphes sont nombreuses et très diversifiées: surfaces isodoses, étalonnages de dispositifs de mesure, essais de détection du rayonnement d'un contaminant, etc. Chaque type d'utilisation nécessite des conditions de travail différentes, et les caractéristiques exigées du mannequin sont, par conséquent, différentes. Aussi, n'est-il pas facile de proposer des critères de comparaison qui soient utilisables dans tous les cas et il serait vain d'y prétendre sur la base des seuls résultats présentés ici. On peut, tout au plus, relever des critères très géné- 
raux d'une part (voir Tableau V), et d'autres, très particuliers, d'autre part, puisqu'ils s'appliquent à l'activation du sodium par irradiation neutronique à l'aide de l'assemblage CRAC.

TABLEAU $\mathrm{V}$

CRITERES GENERAUX DE COMPARAISON

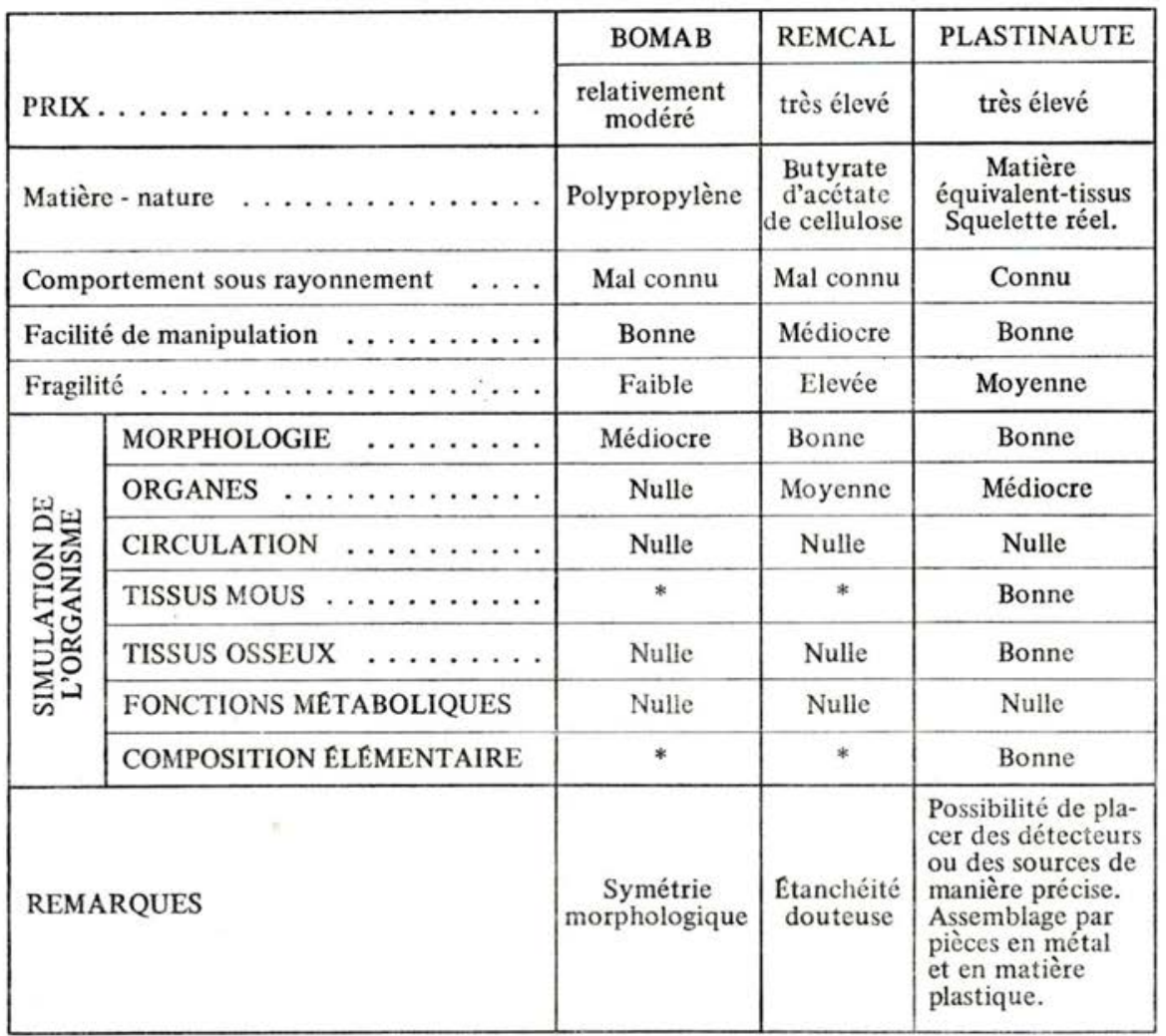

* Dépend de la composition chimique de la solution de remplissage.

Dans ce cas précis, un moyen commode de comparaison est d'évaluer la concordance des valeurs trouvées entre les trois mannequins.

\section{A. IRRADIATION DE FACE}

La surcharge en sodium du Plastinaute conduit à des activités supérieures d'un facteur 2 environ aux valeurs mesurées sur le BomAB et le REMCAL. L'activation moyenne de ces derniers est respectivement de 1,86 $\mu \mathrm{Ci}$ et $1,65 \mu \mathrm{Ci}$ par $\mathrm{g}$ de sodium à $7 \mathrm{~m}$ de la source et pour $10^{18}$ fissions. 


\section{B. Activation Relative SELON L'ORIENTATION}

En prenant comme référence l'activité totale du ${ }^{24} \mathrm{Na}$, mesurée lorsque le mannequin est irradié de face, on constate une bonne concordance pour les trois mannequins, quelle que soit l'orientation. $\mathrm{Si}$ les mannequins se trouvent de profil, on constate une diminution de l'activité totale d'environ $15 \mathrm{p}$. cent à $7 \mathrm{~m}$ de la source).

C. VARIATIONS RElatives de L'ACTIVITÉ SPÉCIFIQUe DANS LES COMPARTIMENTS HOMOLOGUÉS

Ces résultats ne peuvent pas être obtenus de manière directe sur le Plastinaute ; ils concernent les mannequins REMCAL et BomAB.

1. Compartiments en regard de la source

On constate une bonne concordance entre les valeurs obtenues sur les deux mannequins.

\section{Compartiments non en regard de la source}

On constate une divergence qui montre le rôle important joué par les neutrons diffusés et par la morphologie même du mannequin.

\section{ORGANES}

Seul le Remcal permet une mesure fine de l'activité spécifique du ${ }^{24} \mathrm{Na}$ induit dans les organes profonds.

Pour une mesure à $10 \mathrm{p}$. cent près de l'activité totale du ${ }^{24} \mathrm{Na}$ induit par irradiation neutronique, les trois types de mannequin peuvent être employés indifféremment. REMCAL.

L'étude d'organes viscéraux particuliers devrait faire préférer le

Le Plastinaute, de part sa forme et sa constitution, peut constituer un outil de premier ordre pour étudier l'écran ou le réflecteur que constitue le corps humain en matière de criticité.

\section{REMERCIEMENTS}

Nous tenons à remercier ici MM. Chivot, Boulay et Ventadour d'avoir bien voulu mettre leur mannequin REMCAL à notre disposition.

\section{BIBLIOGRAPHIE}

[1] BAILEY J.C. - Criticality accident dosimetry studies. Rapport K-1618 (Oak Ridge National Laboratory), 1964. 
[2] Comar D., Rivière R., Raynaud C., Kellershohn C. - Recherches préliminaires sur la composition et le métabolisme de l'os étudiés par radioactivation neutronique « in vivo * chez l'Homme. Strahlentherapie, 1968, Sonderb. 67, 186-194.

[3] Edelmln I.S., James A.H., Baden H., Moore F.D. - Electrolyte composition of bone and the penetration of radiosodium and deuterium oxide into dog and human bone. J. Clin. Invest., 1954, 33, 192.

[4] Estournel R., Henry P., Beau P., Ergas A. - Evaluation rapide de la dose de neutrons à la suite d'un accident de criticité par mesure de l'activité de ${ }^{24} \mathrm{Na}$. Rapport CEA-R-3083, 1966.

[5] GROUPE DE TRAVAIL \& DOSIMÉTRIE INTERNE 》. - Utilisation d'un fantôme équivalent-tissus pour l'estimation de la contamination radioactive et de la dose absorbée au niveau de certains organes. Radioprotection, 1970, 5, 167.

[6] Hankins D.E. - A study of selected criticality dosimetry methods. Rapport LA-3910, 1968.

[7] Hine G.J., JAGger P.J., BURRows B.A. - Use of a clinical body counter for long term exchangeable sodium studies. In: Whole-body counting, Vienna, IAEA, 1962, vol. 1.

[8] HURST G.S., RITCHIE R.H. - Radiation accidents : dosimetric aspects of neutron and gamma ray exposures. Rapport ORNL-2748, 1959.

[9] McGuIRE S.A. - Neutron activation probability for sodium in man. Rapport LA-3721, 1966.

[10] Morawex T. - Détermination de l'activité ${ }^{24} \mathrm{Na}$ et de l'autodose gamma à partir du spectromètre SNAC. In : Neutron monitoring for radiological protection, Vienna, 29 Aug.-2 Sept., 1966.

[11] Novat J., Revol H., Roussel G. - Communication personnelle (Août 1967).

[12] SANDERS F.W., Auxier J.A. - Neutron activation of sodium in anthropomorphous phantoms. Health Physics, 1962, 8, 371 .

[13] Sмiтh J.M., Boot S.J. - The variation of neutron dose with depth in a tissue equivalent phantom. Phys. Med. Biol., 1962, 7, 45.

[14] Smith J.W. - Sodium activation by fast neutrons in man phantoms. Rapport AERE-R-3697, 1962.

[15] SNyder W.S., Neufeld J. - Calculated depth dose curves in tissue for broad beams of fast neutrons. Brit. J. Radiol., 1955, 28, 342.

[16] Ventadour J. Labat C., Chivot J.J. - Evaluation d'une irradiation neutronique individuelle par l'étude de ses stigmates biologiques. Rapport CEA-R-3884, 1969. 